\title{
Effects of Aging on Angiogenic and Muscle Growth-Related Factors in Naturally Aged Rat Skeletal Muscles
}

\author{
Hyo-Seong Yeo ${ }^{1}$, Jae-Young Lim ${ }^{1}$, Na-Young Ahn ${ }^{2}$ \\ ${ }^{1}$ Department of Rehabilitation Medicine, Seoul National University College of Medicine, Seoul National University Bundang Hospital, Seongnam, Korea \\ ${ }^{2}$ Department of Physical Education, College of Physical Education, Keimyung University, Daegu, Korea
}

Corresponding Author:

Na-Young Ahn, PhD

Department of Physical Education,

College of Physical Education,

Keimyung University, 1095

Dalgubeuldaero, Dalseo-gu, Daegu

42601, Korea

E-mail: nyahn13@kmu.ac.kr

ORCID:

https://orcid.org/0000-0002-7300-5174

Received: November 12, 2020

Revised: December 10, 2020

Accepted: December 11, 2020
Background: This study explored the effects of aging on the expression of angiogenic and muscle protein synthesis factors, as well as the number of satellite cells affecting sarcopenia in naturally aged rat skeletal muscles. Methods: We divided 16 Sprague-Dawley rats into young (12 weeks old, $n=8$ ) and old (24 months old, $n=8$ ) groups and compared muscle and body weight (BW) between them. We also analyzed the expression levels of angiogenic and muscle growth proteins in soleus (slow-twitch) and extensor digitorum longus (EDL; fast-twitch) muscles by western blotting and assessed the number of skeletal muscle satellite cells and myonuclei and mean fiber cross-sectional area (CSA) using by immunofluorescence staining. Results: EDL/BW was significantly lower in old rats than in young rats $(p=0.002)$. The vascular endothelial growth factor level in soleus muscles was significantly lower in old rats than in young rats $(p=0.001)$. Hypoxia-inducible factor 1-alpha and fetal liver kinase 1 levels in EDL muscles were lower in old rats than in young rats $(p=0.001)$. The mammalian target of rapamycin (mTOR), p70S6K, and 4E-BP1 levels were significantly lower in the soleus muscles of old rats than in those of young rats $(p<0.01)$. Similarly, insulin growth factor-1, Akt, mTOR, and p70S6K levels were significantly lower in EDL muscles of old rats than in those of young rats $(p<0.01)$. Additionally, myonuclei/fiber, Pax7/fiber, and mean fiber CSAs in both muscle types were significantly lower in old rats than in young rats $(p<0.01)$. Conclusion: These data suggest different regulation of indices of angiogenic and muscle growth with aging in different muscle types.

Key Words: Aging, Angiogenesis, Protein synthesis, Muscle type, Vascular endothelial growth factor A, mTOR

\section{INTRODUCTION}

Aging exacerbates muscle function, power, strength, and endurance; it also increases the incidence of falls and chronic metabolic diseases, which impact the quality of life. The reduction of muscle function in older adults is predominantly because of the decrease in muscle mass during aging, a process known as sarcopenia. ${ }^{1)}$ As aging progresses, skeletal muscles show decreased responsiveness to physical activity and other anabolic stimuli, resulting in a decrease in the muscle protein synthetic response referred to as anabolic resistance. $^{2)}$
The aberrant expression of proteins involved in muscle cell synthesis and degradation has been implicated in anabolic resistance induction. ${ }^{2)}$ Additionally, protein synthetic response impairments may play key roles in the reduction of muscle mass with aging. ${ }^{3)}$ Age-related changes in anabolic resistance and muscle growth vary among muscle fiber types. ${ }^{4)}$ In humans, the number of muscle fibers, especially in fast-twitch muscles, decreases significantly during aging. ${ }^{5)}$ The mammalian target of rapamycin (mTOR) plays an essential role in muscle protein synthetic responses. $\left.{ }^{6}\right) \mathrm{Al}$ though different mTOR expression levels in different muscle types (slow vs. fast-twitch muscle) in aging animals have been reported, ${ }^{7)}$ 
the study focused only on muscle synthetic protein expression. Recently, mTOR was shown to regulate the proliferation and differentiation of extensor digitorum longus (EDL; fast-twitch) muscle satellite cells in mTOR knockout mice (6 weeks of age). $\left.{ }^{8}\right)$ During aging, the number of satellite cells decreases by approximately $50 \% .^{9)}$ However, little is known about aging-related satellite cell reduction with mTOR-related signaling in different muscle types (slow vs. fast-twitch).

Damage to muscle fiber perfusion owing to aging has recently been suggested to be an important factor driving anabolic resistance. ${ }^{10)}$ Sufficient perfusion of muscle tissue is required to maintain or increase muscle mass, and capillarization is essential for the transportation of oxygen and nutrients to peripheral muscles. Thus, angiogenesis is pivotal for the supply of sufficient oxygen and nutrients to growing muscle fibers. ${ }^{11)}$ Vascular endothelial growth factor (VEGF) is crucial for the formation of new capillaries in adult skeletal muscles. Its expression is regulated by hypoxia-inducible factor-1 alpha (HIF-1 $\alpha)^{12)}$ and decreases in aging skeletal muscle. ${ }^{13)}$ A previous study reported suppressed VEGF protein levels in older adults, with no differences according to the muscle fiber type. ${ }^{14)}$ Additionally, studies have shown that VEGF is essential for muscle protein synthetic responses in skeletal muscle cells. Notably, VEGF depletion in rats (4 months of age) significantly reduced plantaris muscle weight. ${ }^{15)}$ Thus, the aging-induced downregulation of VEGF and subsequent decrease in angiogenesis are considered to be key factors in the induction of anabolic resistance. However, few studies have reported regarding changes in VEGF and mTOR in different muscle types in aged animals, especially in those that naturally aged without specific treatment.

Previous studies on the relationships between VEGF, mTOR, and satellite cells used genetically modified experimental animals, ${ }^{8,15)}$ with limited information on aged animals. Therefore, it is necessary to directly examine aging-related changes occurring in naturally aged individuals. In addition, given that the density and number of capillaries differ depending on the muscle fiber type, the comprehensive exploration of aging-related changes in satellite cell number and mTOR level according to muscle type (slowtwitch vs. fast-twitch) is needed. Hence, we investigated the muscle content and basal phosphorylation of these molecules in soleus (slow-twitch) and EDL (fast-twitch) muscles. We hypothesized that the aging-induced reduction in angiogenic factors would be accompanied by muscle growth factors in different skeletal muscle types of naturally aged rats.

\section{MATERIALS AND METHODS}

\section{Materials}

We divided 16 Sprague-Dawley rats (Samtako, Osan, Korea) into young ( 3 months, $n=8)$ and old (20-24 months, $n=8)$ groups. Two rats per cage were maintained at $22^{\circ} \mathrm{C} \pm 2^{\circ} \mathrm{C}$ and $50 \%-60 \%$ humidity with a $12 / 12$-hour light/dark cycle. The rats were fed with a diet (Samtako) containing $67.5 \%$ carbohydrates, $11.7 \%$ fat, and $20.8 \%$ protein. The animals' dietary intake and body weight (BW) were monitored twice weekly, and we excluded rats exhibiting abnormal symptoms during breeding. All experimental procedures and research methods were approved by the Animal Experiment Ethics Committee of Korea National Sport University (No. KNSU-IACUC-2017-07). The experimental animals' characteristics are presented in Table 1.

\section{Rotarod Test}

Motor coordination was assessed using a rotarod system (B.S. TechnoLab, Seoul, Korea). The rats were allowed to adapt to the equipment at a rate of 4-10 rpm starting 2 days before the test. Motor coordination was tested at $10 \mathrm{rpm}$ for 2 minutes. The duration and number of times that the rats fell from the bar were recorded, as described previously. ${ }^{16)}$

\section{Muscle Sampling}

To exclude the effect on diet, feed was removed except for water 8 hours before euthanize. The rats were anesthetized by injecting an

Table 1. Animal characteristics

\begin{tabular}{lcc}
\hline & Young $(\mathrm{n}=8)$ & Old $(\mathrm{n}=8)$ \\
\hline Body weight (g) & $427.71 \pm 22.21$ & $694.71 \pm 88.13$ \\
Soleus (g) & $0.18 \pm 0.02$ & $0.25 \pm 0.05$ \\
Soleus/BW (\%) & $0.04 \pm 0.01$ & $0.04 \pm 0.01$ \\
EDL (g) & $0.19 \pm 0.02$ & $0.20 \pm 0.06$ \\
EDL/BW (\%) & $0.05 \pm 0.01$ & $0.03 \pm 0.01$ \\
Rotarod test (s) & $79.20 \pm 12.90$ & 0.699 \\
\hline
\end{tabular}

Values are presented as mean \pm standard deviation.

BW, body weight; EDL, extensor digitorum longus. 
anesthetic (xylazine $8 \mathrm{mg} / \mathrm{kg}$ and ketamine $40 \mathrm{mg} / \mathrm{kg}$ ) into the abdominal cavity after 1 week of adaptation. Soleus (slow-twitch) and EDL (fast-twitch) muscle samples were obtained and rapidly frozen in liquid nitrogen or stored at $-80^{\circ} \mathrm{C}$ after embedding in optimal cutting temperature (OCT) compound.

\section{Immunofluorescence}

Immunofluorescence was performed to assess the number of skeletal muscle satellite cells. OCT compound-embedded muscle tissues were cross-sectioned $(10 \mu \mathrm{m})$ at $-20^{\circ} \mathrm{C}$ using a microtome (CM1850; Leica, Wetzlar, Germany). After drying, the muscle tissues were rinsed in T-PBS (0.1\% Tween-20; $\mathrm{pH} 7.4$ ) for 25 minutes, fixed in $4 \%$ paraformaldehyde for 15 minutes, and blocked with T-PBS for 30 minutes. Then, the tissues were incubated overnight at $4^{\circ} \mathrm{C}$ with mouse monoclonal anti-Pax7 and rabbit polyclonal anti-laminin antibodies (1:500; Dako Ltd., Ely, UK). After washing, the tissues were incubated with $\mathrm{Cy} 3$ anti-mouse IgG (1:500; Jackson ImmunoResearch, West Grove, PA, USA) and FITC-conjugated anti-rabbit IgG (1:200; Sigma-Aldrich, St. Louis, MO, USA) antibodies for 1 hour. We determined the number of myonuclear and satellite cells and the mean fiber cross-sectional area (CSA) using AxioVision software (rel. 4.8; Carl Zeiss Microscopy, Jena, Germany).

\section{Western Blotting}

Muscle tissues were incubated for 30 minutes at $4^{\circ} \mathrm{C}$ in lysis buffer containing $25 \mathrm{mM}$ Tris-Cl (pH 7.5), $250 \mathrm{mM} \mathrm{NaCl}, 5 \mathrm{mM}$ EDTA, $1 \%$ NP-40, $1 \mathrm{mM}$ phenylmethylsulfonyl fluoride, and $5 \mathrm{mM}$ dithiothreitol. The supernatant was collected by centrifugation at $14,000 \mathrm{rpm}$ for 30 minutes. The protein concentration of the supernatant (total cytosol fraction) was measured using the Bio-Rad (Bio-Rad Laboratories, Hercules, CA, USA) protein assay reagent according to the manufacturer's instructions. Equal amounts of proteins were mixed with $2 \mathrm{X}$ sodium dodecyl sulfate (SDS) loading buffer containing $60 \mathrm{mM}$ Tris ( $\mathrm{pH}$ 6.8), 25\% glycerol, $2 \%$ SDS, $14.4 \mathrm{mM} 2$-mercaptoethanol, and $0.1 \%$ bromophenol blue. The samples were boiled at $100^{\circ} \mathrm{C}$ for 10 minutes and centrifuged for 20 minutes at $15,000 \mathrm{rpm}$ and $4^{\circ} \mathrm{C}$. Then, the proteins $(100 \mu \mathrm{g})$ were resolved in a $10 \%$ separating gel (30\% acrylamide, $1.5 \mathrm{M}$ Tris [pH 8.8], 10\% SDS, 10\% ammonium persulfate, tetramethylethylenediamine [TEMED]) and a 5\% stacking gel (30\% acrylamide, $1 \mathrm{M}$ Tris [pH 6.8], 10\% SDS, $10 \%$ ammonium persulfate, TEMED) using a Mini-PROTEAN II apparatus (Bio-Rad). Electrophoresis was performed for approximately 2 hours at $80 \mathrm{~V}$. Then, the proteins were transferred onto polyvinylidene difluoride membranes (Bio-Rad) using a Mini Trans-Blot cell system (Bio$\mathrm{Rad}$ ) according to the manufacturer's instructions; the transfer buffer contained $190 \mathrm{mM}$ glycine, $50 \mathrm{mM}$ Tris base, 0.05\% SDS, and 20\% methanol. Then, the membranes were blocked for $5 \mathrm{~min}$ utes with $5 \% \mathrm{w} / \mathrm{v}$ bovine serum albumin solution $(10 \mathrm{mM}$ Tris base, $\mathrm{HCl}$ [pH 7.6], 0.5 M NaCl, 0.05\% Tween-20). After blocking, the membranes were incubated for 12 hours with primary antibodies (anti-VEGF, anti-HIF-1 $\alpha$, anti-FLK-1, anti-insulin growth factor-1 [IGF-1], anti-Akt, anti-mTOR, anti-eIF4E-binding protein 1 [4E-BP1], anti-P70S6 kinase 1 [p70S6K], and anti- $\alpha$-tubulin; all diluted 1:1000 in blocking solution). After five washes, the membranes were incubated for 90 minutes with secondary antibodies (horseradish peroxidase-conjugated goat anti-rabbit 656120 or horseradish peroxidase-conjugated rabbit anti-goat 811620, diluted 1:5000 in blocking solution; Thermo Fisher Scientific, South San Francisco, CA, USA). After five washes, the signal was developed using Western Blotting Luminol Reagent (SC2048; Santa Cruz Biotechnology, Dallas, TX, USA) and visualized on a Molecular Image ChemiDoc XRS system (Bio-Rad). The amount of protein was calculated using the Quantity One 1-D analytical software (Bio-Rad).

\section{Statistical Analysis}

We performed all experiments in duplicate and presented the average values. Statistical analysis was performed using SPSS version 18.0 (SPSS Inc., Chicago, IL, USA). Differences between groups were analyzed using independent-samples t-tests. The significance level was set to $\mathrm{p}<0.05$.

\section{RESULTS}

\section{Differences in Muscle Weight and Motor Coordination between Young and Old Rats}

The BW of old rats was significantly higher than that of young rats $(p=0.001)$. Although the weight of the EDL muscle did not differ significantly between groups $(p=0.699)$, the soleus weight was significantly greater in old rats than in young rats $(p=0.012)$. The soleus mass normalized to BW did not differ significantly between groups ( $\mathrm{p}=0.082)$, whereas the EDL mass normalized to BW was significantly lower in old rats than in young rats $(p=0.002)$. Motor coordination differed significantly between groups, with old rats exhibiting a significantly earlier first drop from the rotarod bar $(\mathrm{p}=0.001)($ Table 1$)$.

\section{Differences in the Expression of Angiogenesis-Related Proteins in Type I and II Muscles of Young and Old Rats}

We assessed the expression levels of different angiogenesis-related proteins in soleus (slow-twitch) and EDL (fast-twitch) muscles. In the soleus muscle, while HIF-1 $\alpha$ and FLK-1 levels did not differ 
between groups, the VEGF level was significantly lower in old rats than in young rats $(p=0.001)$. In the EDL muscle, VEGF levels did not differ significantly between groups ( $p>0.05)$, while HIF$1 \alpha$ and FLK-1 levels were significantly lower in old rats than in young rats (both $\mathrm{p}=0.001)$ (Fig. 1A, 1B).

\section{Differences in the Expression of Muscle Protein Synthesis- Related Proteins in Type I and II Muscles of Young and Old Rats} In the soleus muscle, IGF-1 and Akt levels did not differ significantly between groups, while phosphorylated mTOR, p70S6K, and 4E-BP1 levels were significantly lower in old rats than in young rats $($ all $\mathrm{p}=0.001)$. In the EDL muscle, phosphorylated IGF-1, Akt, mTOR, and p70S6K levels were significantly lower in old rats than in young rats $($ all $\mathrm{p}=0.001)$, while the phosphorylated $4 \mathrm{E}$ BP1 level did not differ significantly between groups $(\mathrm{p}>0.05)$ (Fig. D).
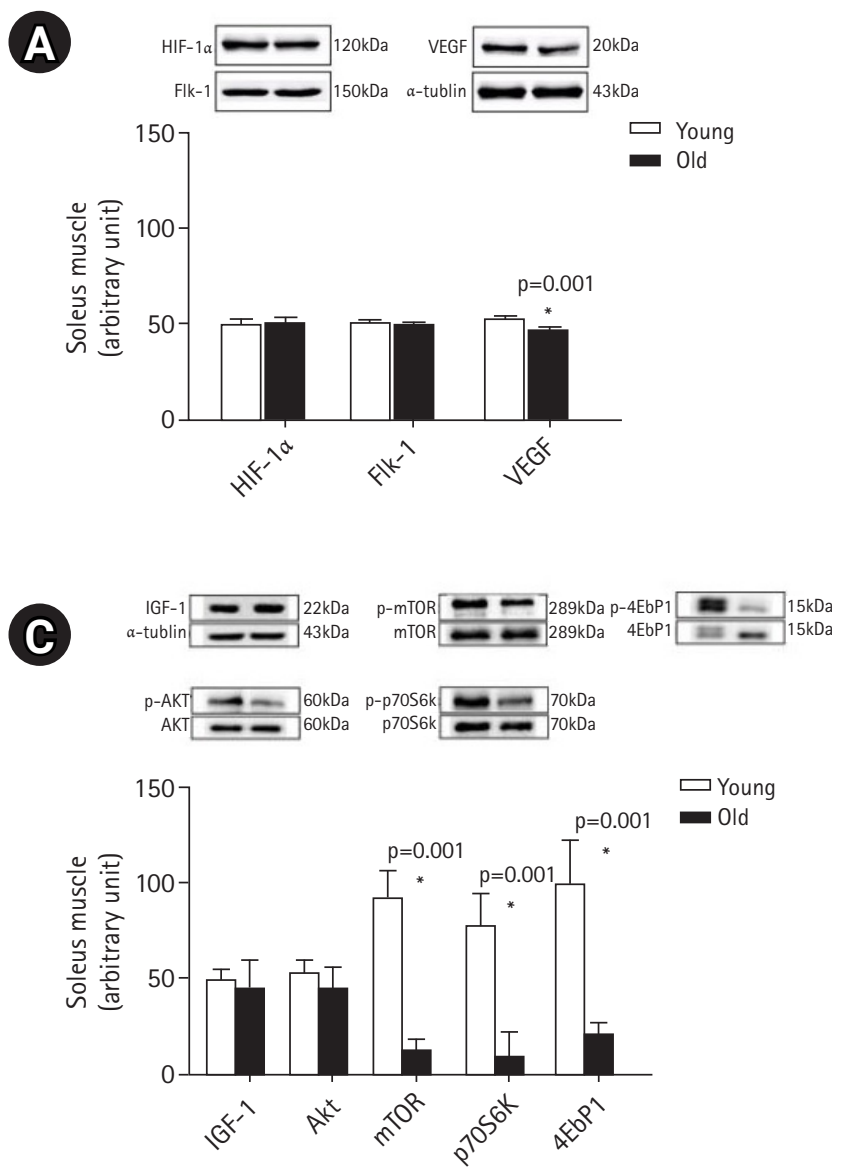

Differences in the Number of Myonuclear and Satellite Cells in Type I and II Muscles of Young and Old Rats

Immunofluorescence analysis revealed significantly lower myonuclei/fiber ( $2.15 \pm 0.25$ vs. $1.66 \pm 0.14)$, Pax7/fiber $(0.04 \pm 0.01$ vs. $0.01 \pm 0.01)$, and muscle $\left(2,169.25 \pm 87.77 \mu \mathrm{m}^{2}\right.$ vs. $1,701.23 \pm 99.85$ $\left.\mu \mathrm{m}^{2} ; \mathrm{p}<0.01\right)$ CSAs in slow-twitch muscles of old rats than in those of young rats. In fast-twitch muscles, myonuclei/fiber $(2.49 \pm 0.55$ vs. $1.32 \pm 0.14), \operatorname{Pax} 7 /$ fiber $(0.14 \pm 0.05$ vs. $0.05 \pm 0.01)$, and muscle $\left(2,277.41 \pm 75.75 \mu \mathrm{m}^{2}\right.$ vs. $\left.1,552.64 \pm 24.61 \mu \mathrm{m}^{2}\right)$ CSAs were significantly lower in old rats than in young rats (all p < 0.01).

\section{DISCUSSION}

This study investigated the effects of aging on the expression of angiogenic and muscle protein synthesis factors, as well as the number of myonuclear and satellite cells, in different skeletal muscle

B

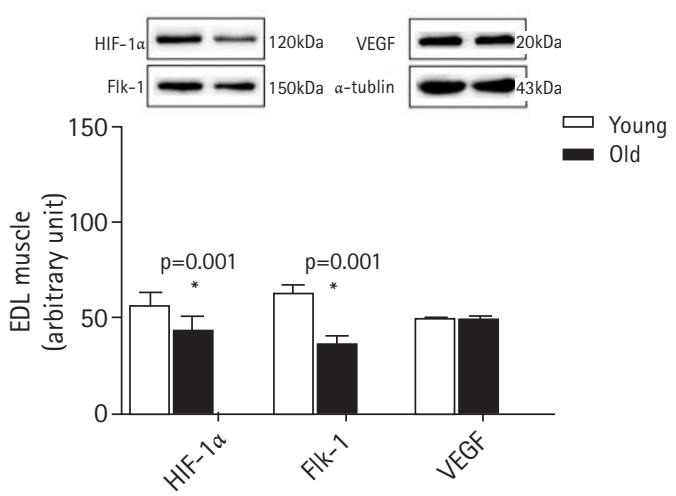

D
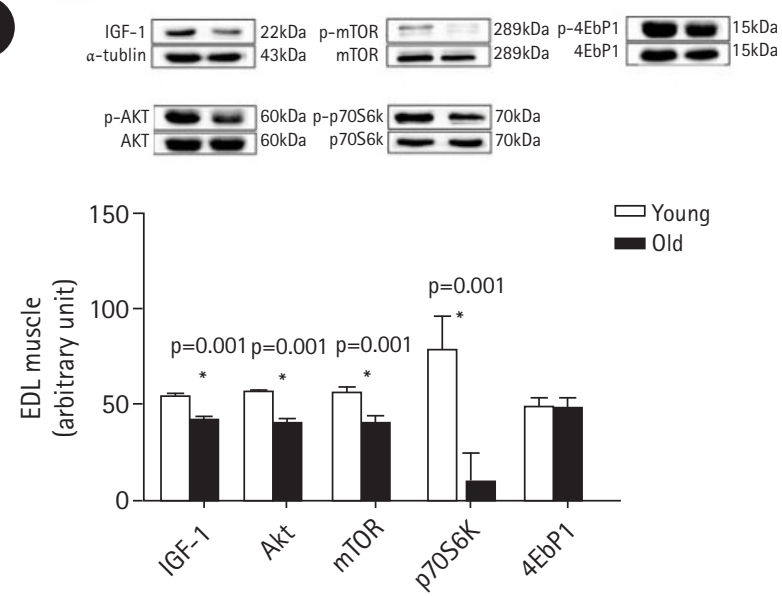

Fig. 1. Expression levels of muscle angiogenic and synthesis-related proteins in slow-twitch and fast-twitch of young $(n=8)$ and old $(n=8)$ rats. (A) Angiogenic protein expressions in soleus muscle. (B) Angiogenic protein expressions in EDL muscle. (C) Muscle synthesis-related protein expressions in soleus muscle. (D) Muscle synthesis-related protein expressions in EDL muscle. EDL, extensor digitorum longus; HIF-1 $\alpha$, hypoxia-inducible factor-1 alpha; VEFG, vascular endothelial growth factor; IGF-1, anti-insulin growth factor-1; mTOR, mammalian target of rapamycin; p70S6K, anti-P70S6 kinase 1; 4E-BP1, anti-eIF4E-binding protein 1. *Significant difference between groups. 
types of naturally aged rats. We found that aging differently reduced the expression of angiogenic and muscle protein synthesis factors in slow-twitch and fast-twitch muscles in naturally aged rats. VEGF expression decreased in soleus but not EDL muscle with aging. In addition, mTOR and p70S6K phosphorylation decreased with aging in both muscle types; however, IGF-1 expression and Akt and 4EBP1 phosphorylation showed different tendencies between muscle types. We also found reduced myonuclear, pax7, and fiber CSAs with age in both muscle types. Thus, reduced angiogenic responses to aging were accompanied by muscle growth responses in naturally aged rat skeletal muscles; however, the mechanisms regulating angiogenic and muscle growth responses appeared to differ according to muscle type (type I vs. type II).

As skeletal muscles predominantly contain microvessels, angiogenesis and adequate muscle tissue perfusion are required to allow sufficient transportation of oxygen and nutrients to peripheral muscles and the maintenance of muscle mass. ${ }^{17)}$ During aging, the angiogenic potential and function of blood vessels considerably decrease because of the reduced production of angiogenic factors. ${ }^{13)}$ VEGF is a $35-45-\mathrm{kDa}$ protein with potent pro-angiogenic effects regulated by HIF-1 $\alpha .{ }^{12)}$ VEGF signals via tyrosine kinase receptors, known as Flk-1/KDR (VEGF receptor-2) predominantly expressed by endothelial cells. ${ }^{18)}$ Importantly, VEGF promotes endothelial cell survival and differentiation and enhances capillary permeability and arteriolar vasodilatation. ${ }^{19)}$ Although the density and number of capillaries in skeletal muscles are known to differ among muscle fiber types, little is known regarding VEGF production in different muscle types. In this study, we observed distinct angiogenic protein expression patterns in slow- and fast-twitch muscles. In slow-twitch muscles (soleus), HIF-1 $\alpha$ levels did not change with aging; however, VEGF levels were 11.4\% lower in old rats than in young rats. This finding suggests that eNOS (endothelial nitric oxide synthase), another indicator that regulates VEGF expression, ${ }^{20)}$ may affect the decreased VEGF level in the soleus muscle. Therefore, the angiogenic response through VEGF signals may differently occur according to the muscle type in naturally aged rats.

The aging-induced decreases in VEGF expression and angiogenesis have been shown to induce anabolic resistance associated with Akt downregulation. In VEGF-deficient mice, the plantaris muscle mass was lower at 30 days after functional overload, while the average muscle fiber area was also lower. The phosphorylation level of Akt was also lower than that of wild-type in the study. ${ }^{15}$ )

mTOR is a protein kinase ${ }^{21)}$ and is sensitive to the activity of IGF-1, a mediator of growth hormone activity. ${ }^{6}$ When IGF-1 binds to the receptor, it activates intracellular phosphatidylinosi- tol-3 kinase/Akt to ultimately activate mTOR. ${ }^{6}$ mTOR activity is involved in protein synthesis through two independent pathways via $\mathrm{p} 70 \mathrm{~S} 6 \mathrm{~K}$ or $4 \mathrm{E}-\mathrm{BP} 1$. Increased $\mathrm{p} 70 \mathrm{~S} 6 \mathrm{~K}$ activity by mTOR activity induces the phosphorylation of the ribosomal protein $\mathrm{S} 6$, which induces protein synthesis, 4E-BP1 inhibits eIF4E activity. ${ }^{22)}$ mTOR activity separates 4E-BP1 from eIF4E to form eIF4F, a complex of eIF4E and eIF4G, and initiates protein synthesis. ${ }^{23)} \mathrm{A}$ study investigating changes in muscle mass and mTOR-related signaling proteins in the soleus and EDL muscles of the F344BN rat model with aging reported that both SOL/BW and EDL/BW decreased with age. In the soleus muscle of old male mice, p-Akt, p-mTOR, and p-p70S6K decreased, whereas in the EDL muscle, p-Akt and p-mTOR increased, while p-p70S6K decreased, showing different results between muscle types. ${ }^{7)}$ Likewise, in our study, we observed partially different protein expression between the soleus and EDL muscles. The soleus/BW tended to decrease with age, although the difference was not statistically significant, and the EDL/BW decreased significantly. We also observed consistently lower mTOR and P70S6K phosphorylation levels in slowand fast-twitch muscles of old rats than in those of young rats. However, IGF-1 and Akt phosphorylation levels were lower in old rats than in young rats, but only in fast-twitch muscle fibers. Although mTOR, the key factor of muscle growth, was downregulated in both muscle types with age, other factors responded differently depending on the muscle types. In addition, contrary to our expectation, we observed VEGF reduction only in the soleus muscle. This finding was consistent with with that of a previous study showing that mTOR regulates VEGF. ${ }^{24)}$ Therefore, we observed a relationship between VEGF and mTOR in slow- but not fasttwitch muscles, indicating that different muscle fiber types may have different muscle growth mechanisms in skeletal muscles of naturally aged rats.

Satellite cells are muscle precursor cells that occupy "satellite" cell positions in relation to skeletal muscle fibers. ${ }^{25)}$ In adult skeletal muscles, quiescent satellite cells are activated upon muscle damage, promoting muscle repair. ${ }^{26)}$ Satellite cells are related to VEGF expression in skeletal muscle. ${ }^{15)}$ In addition, a reduction in the number of satellite cells precedes aging-induced muscle fiber atrophy, ${ }^{27)}$ suggesting that the satellite cell number is a strong predictor of muscle fiber aging. ${ }^{28)}$ Human studies reported no difference in satellite cell content between type I and type II muscle fiber types of young adults; ${ }^{29)}$ however, the satellite cell/fiber of type II fibers were significantly reduced compared to that of type I fibers in older adults. ${ }^{28)}$ In contrast, animal studies reported decreases in the proportions of both soleus and EDL satellite cells with aging. ${ }^{30)}$ In the present study, we found that the satellite cell number decreased with age equivalently in slow-twitch and fast-twitch rat muscles 
Table 2. Number of myonuclear and satellite cells in type I and II muscles of young and old rats

\begin{tabular}{|c|c|c|c|c|c|c|}
\hline & \multicolumn{3}{|c|}{ Soleus } & \multicolumn{3}{|c|}{ EDL } \\
\hline & Young $(\mathrm{n}=8)$ & Old $(n=8)$ & $\mathrm{p}$-value & Young $(\mathrm{n}=8)$ & Old $(n=8)$ & $\mathrm{p}$-value \\
\hline $\operatorname{CSA}\left(\mu \mathrm{m}^{2}\right)$ & $2,169.25 \pm 87.77$ & $1,701.23 \pm 99.85^{*}$ & 0.001 & $2,277.41 \pm 75.75$ & $1,552.64 \pm 24.61^{*}$ & 0.001 \\
\hline Myonuclei/fiber & $2.15 \pm 0.25$ & $1.66 \pm 0.14^{*}$ & 0.008 & $2.49 \pm 0.55$ & $1.32 \pm 0.14^{*}$ & 0.002 \\
\hline $\operatorname{Pax} 7 /$ fiber & $0.04 \pm 0.01$ & $0.01 \pm 0.01^{*}$ & 0.001 & $0.14 \pm 0.05$ & $0.05 \pm 0.01^{*}$ & 0.003 \\
\hline
\end{tabular}

Values are presented as mean \pm standard deviation.

EDL, extensor digitorum longus; CSA, cross-sectional area.

*Significant difference from the young group.

Young

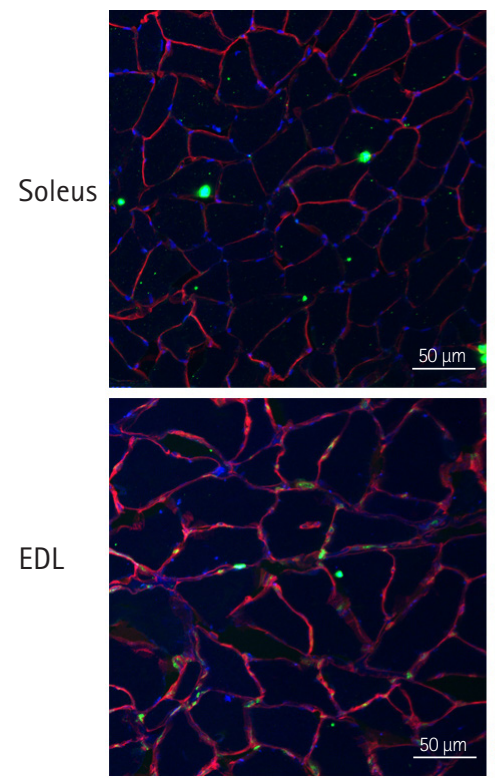

Old
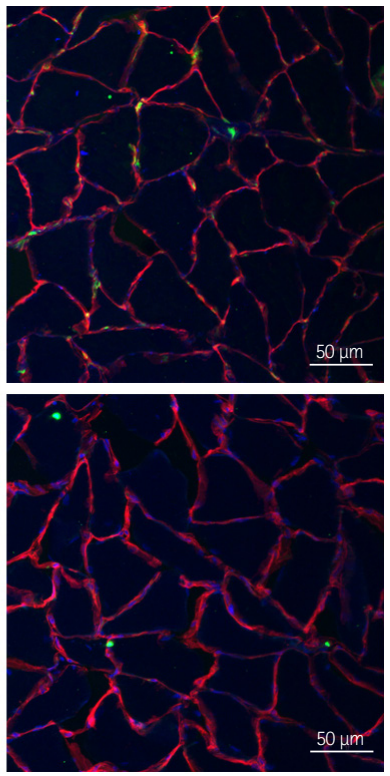

Fig. 2. Representative immunofluorescence images of stained myonuclear and satellite cells in types I and II muscles of young and aged rats (magnification, $\times 20$ ). Red indicates laminin staining; blue, 4',6-diamidino-2-phenylindole (DAPI); and green, Pax 7. EDL, extensor digitorum longus.

(Table 2, Fig. 2). Additionally, we found that VEGF levels in slowtwitch muscles reduced with age. Given that satellite cells produce VEGF, the aging-induced reduction in satellite cell numbers might have contributed to the reduction in VEGF levels.

Although this study provides significant insights on reduced angiogenic and muscle growth factors in skeletal muscles of naturally aged rats, it is important to acknowledge its limitations. First, as the study sample consisted of experimental animals, the results may not generalize to human skeletal muscles. Second, this study used soleus and EDL as slow and fast-twitch muscles; thus, we could not provide data showing diversification of fiber type in each muscle type. Finally, further morphological studies are required to understand the relevance of the proximity of satellite cells to capillaries in skeletal muscles, which might deepen our understanding of the complex rela-

tionship between angiogenesis and muscle growth.

In conclusion, this study investigated factors related to angiogenesis, muscle protein synthesis, and satellite cells in different skeletal muscle types (slow-twitch and fast-twitch) of young and old rats. We found that aging differently impaired angiogenic and muscle growth responses in slow-twitch and fast-twitch muscle fibers. This study is the first to comprehensively examine aging-induced alterations in angiogenesis, muscle protein synthesis, and satellite cell numbers in different muscle types. We believe that a deeper understanding of the muscle growth response according to muscle fiber types is necessary. In addition, in-depth morphological analyses are required to elucidate the spatial relationship between capillaries and satellite cells in aging skeletal muscles.

\section{ACKNOWLEDGMENTS}

\section{CONFLICT OF INTEREST}

The researchers claim no conflicts of interest.

\section{FUNDING}

This work was supported by the Ministry of Education of the Republic of Korea and the National Research Foundation of Korea (No. NRF-2017S1A5B5A01024774). This work was supported by the National Research Foundation of Korea grant funded by the Korea government (MSIT) (No. 800-20200179) and Aging \& Mobility Biophysics Laboratory of Seoul National University Bundang Hospital.

\section{AUTHOR CONTRIBUTION}

Conceptualization, HSY, NYA; Data curation, HSY; Funding acquisition, HSY, NYA; Investigation, HSY; Methodology, HSY; Project administration, HSY, NYA; Supervision, JYL, NYA; Writing-original draft, HSY; Writing-review \& editing, HSY, JYL.

\section{REFERENCES}

1. Jang HC. How to Diagnose Sarcopenia in Korean Older Adults? 
Ann Geriatr Med Res 2018;22:73-9.

2. Rennie MJ. Anabolic resistance: the effects of aging, sexual dimorphism, and immobilization on human muscle protein turnover. Appl Physiol Nutr Metab 2009;34:377-81.

3. Burd NA, Wall BT, van Loon LJ. The curious case of anabolic resistance: old wives' tales or new fables? J Appl Physiol (1985) 2012;112:1233-5.

4. Lambert CP, Evans WJ. Effects of aging and resistance exercise on determinants of muscle strength. J Am Aging Assoc 2002; 25:73-8.

5. Oh SL, Yoon SH, Lim JY. Age- and sex-related differences in myosin heavy chain isoforms and muscle strength, function, and quality: a cross sectional study. J Exerc Nutrition Biochem 2018;22:43-50.

6. Wang X, Proud CG. The mTOR pathway in the control of protein synthesis. Physiology (Bethesda) 2006;21:362-9.

7. Paturi S, Gutta AK, Katta A, Kakarla SK, Arvapalli RK, Gadde MK, et al. Effects of aging and gender on muscle mass and regulation of Akt-mTOR-p70s6k related signaling in the F344BN rat model. Mech Ageing Dev 2010;131:202-9.

8. Zhang P, Liang X, Shan T, Jiang Q, Deng C, Zheng R, et al. mTOR is necessary for proper satellite cell activity and skeletal muscle regeneration. Biochem Biophys Res Commun 2015; 463:102-8.

9. Chakkalakal JV, Jones KM, Basson MA, Brack AS. The aged niche disrupts muscle stem cell quiescence. Nature 2012;490: 355-60.

10. Rasmussen BB, Fujita S, Wolfe RR, Mittendorfer B, Roy M, Rowe VL, et al. Insulin resistance of muscle protein metabolism in aging. FASEB J 2006;20:768-9.

11. Egginton S, Badr I, Williams J, Hauton D, Baan GC, Jaspers RT. Physiological angiogenesis is a graded, not threshold, response. J Physiol 2011;589(Pt 1):195-206.

12. Tang K, Breen EC, Gerber HP, Ferrara NM, Wagner PD. Capillary regression in vascular endothelial growth factor-deficient skeletal muscle. Physiol Genomics 2004;18:63-9.

13. Ryan NA, Zwetsloot KA, Westerkamp LM, Hickner RC, Pofahl WE, Gavin TP. Lower skeletal muscle capillarization and VEGF expression in aged vs. young men. J Appl Physiol (1985) 2006; 100:178-85.

14. Croley AN, Zwetsloot KA, Westerkamp LM, Ryan NA, Pendergast AM, Hickner RC, et al. Lower capillarization, VEGF protein, and VEGF mRNA response to acute exercise in the vastus lateralis muscle of aged vs. young women. J Appl Physiol (1985) 2005;99:1872-9.

15. Huey KA, Smith SA, Sulaeman A, Breen EC. Skeletal myofiber VEGF is necessary for myogenic and contractile adaptations to functional overload of the plantaris in adult mice. J Appl Physiol (1985) 2016;120:188-95.

16. Saputra D, Chang J, Lee BJ, Yoon JH, Kim J, Lee K. Short-term manganese inhalation decreases brain dopamine transporter levels without disrupting motor skills in rats. J Toxicol Sci 2016; 41:391-402.

17. Timmerman KL, Lee JL, Dreyer HC, Dhanani S, Glynn EL, Fry CS, et al. Insulin stimulates human skeletal muscle protein synthesis via an indirect mechanism involving endothelial-dependent vasodilation and mammalian target of rapamycin complex 1 signaling. J Clin Endocrinol Metab 2010;95:3848-57.

18. Hicklin DJ, Ellis LM. Role of the vascular endothelial growth factor pathway in tumor growth and angiogenesis. J Clin Oncol 2005;23:1011-27.

19. He Y, Yu S, Hu J, Cui Y, Liu P. Changes in the anatomic and microscopic structure and the expression of HIF-1 $\alpha$ and VEGF of the yak heart with aging and hypoxia. PLoS One 2016;11:e0149947.

20. Iemitsu M, Maeda S, Jesmin S, Otsuki T, Miyauchi T. Exercise training improves aging-induced downregulation of VEGF angiogenic signaling cascade in hearts. Am J Physiol Heart Circ Physiol 2006;291:H1290-8.

21. Wiederrecht GJ, Sabers CJ, Brunn GJ, Martin MM, Dumont FJ, Abraham RT. Mechanism of action of rapamycin: new insights into the regulation of G1-phase progression in eukaryotic cells. Prog Cell Cycle Res 1995; 1:53-71.

22. Jefferies HB, Fumagalli S, Dennis PB, Reinhard C, Pearson RB, Thomas G. Rapamycin suppresses 5'TOP mRNA translation through inhibition of p70s6k. EMBOJ 1997;16:3693-704.

23. Beugnet A, Wang X, Proud CG. Target of rapamycin (TOR)-signaling and RAIP motifs play distinct roles in the mammalian TOR-dependent phosphorylation of initiation factor 4E-binding protein 1.J Biol Chem 2003;278:40717-22.

24. Wang S, Lu J, You Q, Huang H, Chen Y, Liu K. The mTOR/ AP-1/VEGF signaling pathway regulates vascular endothelial cell growth. Oncotarget 2016;7:53269-76.

25. Mauro A. Satellite cell of skeletal muscle fibers. J Biophys Biochem Cytol 1961;9:493-5.

26. Wagers AJ, Conboy IM. Cellular and molecular signatures of muscle regeneration: current concepts and controversies in adult myogenesis. Cell 2005; 122:659-67.

27. Brack AS, Bildsoe H, Hughes SM. Evidence that satellite cell decrement contributes to preferential decline in nuclear number from large fibres during murine age-related muscle atrophy. J Cell Sci 2005;118(Pt 20):4813-21.

28. Verdijk LB, Gleeson BG, Jonkers RA, Meijer K, Savelberg HH, Dendale P, et al. Skeletal muscle hypertrophy following resistance training is accompanied by a fiber type-specific increase in 
satellite cell content in elderly men. J Gerontol A Biol Sci Med Sci 2009;64:332-9.

29. Kadi F, Charifi N, Denis C, Lexell J, Andersen JL, Schjerling P, et al. The behaviour of satellite cells in response to exercise: what have we learned from human studies? Pflugers Arch 2005;
451:319-27.

30. Gibson MC, Schultz E. Age-related differences in absolute numbers of skeletal muscle satellite cells. Muscle Nerve 1983;6:57480. 